\title{
Reflets
}

Revue ontaroise d'intervention sociale et communautaire

\section{Affirmation politique des femmes au sein du milieu associatif}

\section{Lyne Bouchard}

Volume 3, numéro 2, automne 1997

Visibles et Partenaires : Pratiques et recherches féministes

URI : https://id.erudit.org/iderudit/026180ar

DOI : https://doi.org/10.7202/026180ar

Aller au sommaire du numéro

Éditeur(s)

Reflets : Revue ontaroise d'intervention sociale et communautaire

ISSN

1203-4576 (imprimé)

1712-8498 (numérique)

Découvrir la revue

Citer cet article

Bouchard, L. (1997). Affirmation politique des femmes au sein du milieu associatif. Reflets, 3(2), 187-200. https://doi.org/10.7202/026180ar

Tous droits réservés (C) Reflets : Revue ontaroise d'intervention sociale et communautaire, 1997
Ce document est protégé par la loi sur le droit d'auteur. L'utilisation des services d'Érudit (y compris la reproduction) est assujettie à sa politique d'utilisation que vous pouvez consulter en ligne.

https://apropos.erudit.org/fr/usagers/politique-dutilisation/ 


\section{Affirmation politique des femmes au sein du milieu associatif}

\section{Lyne Bouchard}

Responsable du comité de démarche politique de la Table féministe francophone de concertation provinciale de l'Ontario ${ }^{1}$

\section{Introduction}

Comme représentante de la Table féministe francophone de concertation provinciale de l'Ontario, j'ai oeuvré durant plus de deux ans et demi au sein de la Coalition pour le développement et l'épanouissement de la communauté franco-ontarienne et des minorités raciales francophones de l'Ontario. C'est de cette expérience dont je voudrais vous entretenir dans le cadre de cet article. Consciente qu'il s'agit d'un élément dans une dynamique politique beaucoup plus large où la Table féministe est engagée activement, je crois cependant que cette expérience est riche de leçons pour l'avenir. En effet, la création de la Coalition, en juin 1994, a été un événement important dans l'histoire du milieu associatif francophone en Ontario. C'est pourquoi j'ai voulu prendre l'exemple de la participation des femmes à cet espace de concertation pour mieux montrer les enjeux liés à l'affirmation politique des femmes.

Avant d'aborder cette expérience, je ferai, dans un premier temps, un bref retour historique sur la création de la Table féministe. Je me pencherai principalement sur son modèle de leadership et sa membriété, car ce sont ces éléments qui ont guidé nos interventions au sein de la Coalition. Dans un second temps, je présenterai le contexte politique et les objectifs poursuivis par 
notre participation à la Coalition et j'aborderai les principales stratégies internes et externes pour faire avancer la vision des féministes au sein de cette Coalition. Finalement, je lancerai quelques pistes de réflexion pouvant servir à établir les futures stratégies de participation des femmes et de la Table féministe au débat sur l'avenir du leadership en Ontario français.

\section{Historique de la Table féministe francophone de concertation provinciale de l'Ontario}

Cette année, nous fêtons en quelque sorte le cinquième anniversaire de la création de la Table féministe. En effet, c'est lors du premier colloque sur l'intervention féministe en Ontario qui a eu lieu en février 1992, à Sudbury, que fut créé cet organisme. À ce rendez-vous féministe, les femmes présentes ont voulu se donner une voix politique. Elles ont alors mandaté quelques femmes pour voir à la mise en place éventuelle d'un organisme féministe ayant un mandat politique clair et une structure organisationnelle souple, flexible et accessible. Intuitivement, les femmes avaient eu, dès le départ, une certaine sagesse, celle d'éviter une structure lourde, coûteuse et pouvant rapidement devenir sclérosée.

Les premières rencontres, réunissant les représentantes de groupes pressentis pour prendre part à la Table féministe, ont permis d'établir le plan d'organisation. Le principe selon lequel les groupes devaient d'abord apprendre à se connaître, à se comprendre, voire à mieux s'organiser avant de participer au débat public, a guidé nos premiers pas. Plus que tout, nous voulions d'abord être un regroupement qui serait un lieu de concertation entre nous, les groupes de femmes, puis ensuite, un groupe d'action politique. Nous avons donc pris le temps nécessaire pour établir des liens de confiance. Nous nous sommes posées toutes sortes de questions propres à favoriser notre développement comme groupe. Au moyen d'ateliers, nous avons clarifié notre mandat, 
«La Table féministe est devenue ainsi une tribune féministe provinciale dont le mandat principal est la concertation entre groupes et l'action politique.» défini notre mode de fonctionnement et délimité certains dossiers de travail.

La Table féministe est devenue ainsi une tribune féministe provinciale dont le mandat principal est la concertation entre groupes et l'action politique.

Elle est féministe car elle agit en vue d'une société juste, saine et équitable qui reconnaît les expériences et les valeurs de toutes les femmes, leurs diversités, leurs spécificités et qui luttent contre toutes les formes de discrimination.

La Table féministe vise la concertation et l'action politique car elle est un lieu où des groupes, d'un commun accord, partagent et échangent leurs connaissances dans la diversité, créent des solidarités et développent des stratégies de revendications, de pressions et d'interventions politiques.

La Table féministe est provinciale car elle est constituée de groupes oeuvrant en Ontario français et son action a aussi une portée nationale et internationale ${ }^{2}$.

Ce long travail de construction du groupe peut avoir nui, de prime abord, à la visibilité de la Table féministe au plan politique, car elle n'intervenait pas, par choix, durant ce temps sur les dossiers chauds de l'actualité, pour se faire connaître des médias et de la population, voire des autres groupes du milieu. Les représentantes ont jugé préférable de prendre le temps de renforcer les solidarités avant de partir à la conquête de l'espace public.

Au départ, les groupes membres de la Table féministe étaient exclusivement des groupes de femmes francophones. Toutefois, nous avons voulu que des comités autonomes de femmes existant déjà au sein des groupes mixtes francophones fassent partie de la Table féministe. C'est ainsi qu'au fil du temps, ces comités ${ }^{3}$ ou des comités francophones existants à l'intérieur de groupes de femmes anglophones sont également devenus membres de la Table féministe. Les raisons qui ont dicté cet élargissement de la membriété sont fort simples. Nous souhaitions que les groupes 
"La Table féministe a adopté, dès sa création, une structure-culture organisationnelle transversale dans son fonctionnement et sa représentation.»

«Le mode de représentation et de leadership s'appuie essentiellement sur les expertises de chacune." francophones mixtes et les groupes anglophones incluent respectivement la composante femme ou francophone à la structure officielle de leur organisme. Pour les instigatrices de la Table féministe, cette politique permet une visibilité et une reconnaissance tant de la francophonie dans un milieu majoritairement anglophone que des questions féministes au sein du milieu francophone. Par ailleurs, quelques membres siègent à la Table féministe si, de par le milieu qu'elles représentent ou leur formation, elles apportent un élément de diversité ou une expertise absente au sein de la Table féministe.

La Table féministe a adopté, dès sa création, une structureculture organisationnelle transversale dans son fonctionnement et sa représentation. Plusieurs comités, se penchant sur des dossiers ou sur le mode de fonctionnement ont été mis sur pied. Ils sont autonomes et ont une responsable qui coordonne l'ensemble de ses activités. Depuis la dernière année, un comité de coordination, composé de cinq femmes ne participant à aucun autre comité, voit à la bonne marche de l'ensemble des activités et aux imprévus pouvant survenir entre les rencontres générales de la Table féministe qui ont lieu deux fois l'an. L'information circule d'un comité à l'autre et d'une rencontre générale à l'autre. L'influence est également transversale car chaque comité détermine l'orientation de ses dossiers, tout en tenant compte des préoccupations exprimées par les autres comités. Par ailleurs, les porte-parole de la Table féministe varient selon les dossiers, les expertises et les disponibilités de chacune. Le mode de représentation et de leadership s'appuie essentiellement sur les expertises de chacune. Cela a comme résultat que plusieurs femmes interviennent publiquement et simultanément sur les différents dossiers de l'heure. Cette forme de leadership cherche à partager davantage le pouvoir entre les femmes. Elle favorise également la formation et le mentorat entre pairs comme moyen d'action pour les femmes. Cette formation est informelle et se concrétise souvent en participant aux activités ou aux comités internes comme aux activités externes à la Table féministe.

Après cinq ans de travail dans ce cadre organisationnel, nous constatons pourtant certains inconvénients liés à cette culture 
transversale de fonctionnement et de leadership. En effet, les responsables de dossiers ou de comités semblent être nommées à vie. La tâche qui leur incombe est lourde. De plus, les responsables ne reçoivent aucune rémunération pour leur travail. Ces deux aspects en font hésiter plusieurs lorsque vient le temps de songer à une relève. Présentement, nous nous penchons sur cette question afin d'y apporter une solution.

\section{Coalition pour le développement et l'épanouissement de la communauté franco-ontarienne et des minorités raciales francophones de l'Ontario}

En juin 1994, Patrimoine Canada, un ministère du gouvernement fédéral, organise un forum communautaire réunissant les groupes francophones de l'Ontario qui reçoivent des subventions dans le cadre du Programme d'appui aux langues officielles et à la coordination des articles 41 et 42 de la Loi sur les langues officielles. Lors de ce forum, Patrimoine Canada propose d'établir de nouvelles règles de financement des groupes dans le cadre de ce qu'il nomme l'Entente Canada-Communauté, région Ontario. Ces nouvelles règles accordent aux groupes francophones une participation accrue dans la répartition de l'enveloppe budgétaire rattachée au Programme. En fait, Patrimoine Canada avait élaboré cinq propositions comportant chacune des niveaux différents de participation et les groupes devaient choisir celle qui leur convenait le mieux. Pourtant, les représentantes et représentants des groupes présents ont vu, dans ces propositions, une manoeuvre du Ministère pour amener les groupes à gérer eux-mêmes la décroissance appréhendée du financement. La communauté franco-ontarienne a donc refusé le processus proposé par Patrimoine Canada en invoquant que tout avait été décidé d'avance, que personne n'avait été consulté et qu'une des propositions reprenait l'idée d'un Plan global de financement, proposition qui avait déjà été rejetée par 
la communauté quelques années auparavant. C'est à cette occasion qu'est née la Coalition ${ }^{4}$.

La Coalition a pour mandat de négocier une entente de cogestion avec Patrimoine Canada. De janvier à juin 1995, l'exécutif de la Coalition, formé d'une vingtaine de groupes et de leurs représentantes et représentants, a élaboré un modèle de cogestion et a jeté les bases d'un modèle de concertation. Ce modèle a fait l'objet de négociation avec Patrimoine Canada qui l'a accepté. Un troisième forum communautaire, réunissant l'ensemble des groupes membres de la Coalition, a eu lieu en juin 1995. À cette occasion, on a entériné, avec quelques modifications, le modèle et l'Entente intervenue avec Patrimoine Canada, puis on a reconduit le mandat de la Coalition et de son exécutif.

\section{Pourquoi la Table féministe se joint-elle à l'exécutif de la Coalition?}

«Pour la première fois de l'histoire de l'Ontario français, les femmes décident de s'asseoir à la même table que les principaux définisseurs de situation du milieu associatif francophone.»
Diverses raisons liées au contexte historique, politique et structurel ainsi que des objectifs précis ont motivé la participation de la Table féministe à la Coalition.

\section{Contexte}

Pour la première fois de l'histoire de l'Ontario français, les femmes décident de s'asseoir à la même table que les principaux définisseurs de situation du milieu associatif francophone ${ }^{5}$. Et elles décident d'y participer en vue d'élaborer un projet commun. Cette initiative, à elle seule, constitue un fait tournant. Ainsi, en marge de la négociation de l'Entente Canada-Communauté, la Coalition a dû s'atteler à la tâche de repenser le monde associatif de la francophonie ontarienne. Cette réflexion a porté sur deux aspects précis : «'établissement des mécanismes de représentation politique et la détermination des personnes qui formeront le Comité d'appui à la communauté dans le cadre de l'Entente» ${ }^{6}$. Il s'agissait, en somme, 
«Elle a énoncé

clairement que sa participation à la Coalition avait pour principal objectif celui de l'équité.» de déterminer les porte-parole officiels et les agents de liaison de la communauté francophone de l'Ontario auprès de Patrimoine Canada.

La structure retenue correspond d'assez près au modèle vécu et préconisé par la Table féministe 7 . De plus, ce genre d'affiliation de partenaires provenant de plusieurs secteurs était une première en Ontario français.

\section{Objectifs poursuivis}

Dès la première rencontre, la Table féministe a indiqué les raisons de son affiliation comme partenaire et les motifs de sa participation à l'exécutif de la Coalition ${ }^{8}$. Elle a énoncé clairement que sa participation à la Coalition avait pour principal objectif celui de l'équité. Les femmes qui se sont succédé pour représenter la Table féministe à la Coalition ont toujours su réitérer cet objectif. Au fil des mois, cet objectif a pris corps. Nos interventions répondaient à la nécessité de doter le milieu associatif franco-ontarien de principes et de pratiques de travail plus équitables tant au niveau de la représentation publique et politique qu'au niveau du financement, du développement et de l'épanouissement des communautés francophones.

En cours de route, un second objectif s'est ajouté, soit celui de la parité.

La Table féministe a présenté le dossier comme un principe qu'elle souhaite voir étendre à l'ensemble $d u$ milieu associatif de l'Ontario français. Depuis les débuts de la Coalition, la Table féministe a toujours affirmé qu'à l'instar de [sic]d'autres groupes de la communauté, elle est dorénavant prête à assumer un plus grand leadership et une représentation accrue au sein de la francophonie ontariennes.

Nous avons donc travaillé et réussi à obtenir, dans les clauses de l'Entente, la parité dans toutes les structures décisionnelles. L'atteinte d'un tel objectif est le fruit des stratégies adoptées par la Table féministe au sein de la Coalition. 


\section{Stratégies générales}

"...une telle stratégie de prise de parole conteste directement les habitudes de la chose publique et politique de la gent masculine de l'Ontario français..."
J'envisagerai ici les deux principales stratégies adoptées par la Table féministe à la Coalition, soit la prise de parole et la création d'alliances avec certains partenaires.

\section{Prise de parole}

La première stratégie, et la plus souvent utilisée, a été la prise de parole. Elle consiste à nommer les problèmes, les oppositions, voire les conflits d'intérêts existant entre certains «partenaires». Cette stratégie a été employée à la fois pour affirmer les objectifs poursuivis par la Table féministe, pour dénoncer les traitements de faveur et les privilèges dont bénéficiaient certains membres de la Coalition, ou pour dévoiler les intérêts d'autres groupes en présence. Ainsi, à l'intérieur de la Coalition, la prise de parole de la représentante de la Table féministe a pris la forme d'un questionnement continu sur les intentions des représentantes et représentants de certains organismes, de demandes de clarifications, de rappels constants de l'objectif du travail de la Coalition et des principes qu'elle poursuit, d'identification des apparences de conflits d'intérêts éventuels ou de dénonciation de conflits d'intérêts avérés.

Cette stratégie a été vivement contestée par une majorité de représentantes et représentants d'autres associations, ce qui a résulté, plus souvent qu'autrement, en l'isolement de la représentante de la Table féministe au sein de la Coalition, et même parfois, en des insultes personnelles à peine déguisées. Il va sans dire qu'une telle stratégie de prise de parole conteste directement les habitudes de la chose publique et politique de la gent masculine de l'Ontario français (les définisseurs de situation) et ce depuis l'époque de la Patente à aujourd'hui ${ }^{10}$.

Par ailleurs, soulignons qu'une prise de parole, extérieure à la Coalition, a été entreprise par la Table féministe avec le concours, entre autres, des médias et de fonctionnaires. 


\section{Alliances}

En raison d'une vision et d'une expérience commune de leadership, une affinité très forte s'est développée entre la Table féministe et l'Alliance culturelle de l'Ontario. Plusieurs positions défendues par la Table féministe à la Coalition ont été prises de concert avec l'Alliance.

Les relations entre la Table féministe et les autres groupes ont été plus circonstancielles. La Table féministe a ainsi obtenu leur appui sur les principes d'équité et de parité, notamment en ce qui a trait à l'élaboration du texte de l'Entente.Toutefois, lorsqu'est venu le temps de préciser les mécanismes d'application de ces principes dans la distribution de fonds et la représentation politique, cette belle unanimité s'est vite effritée. Nous avons alors perçu l'ampleur des enjeux et des contradictions liés à ces questions. Bien entendu, le financement de chacun de leur groupe était important, mais leur affiliation à l'Association canadienne-française de l'Ontario provinciale semblait tout aussi problématique. En effet, la transformation des mécanismes de distribution de fonds et de représentation politique pouvait sérieusement miner le financement et le rôle traditionnel de porte-parole de l'ACFO provinciale. Dès lors, il nous est apparu impossible que les sections régionales de l'ACFO et les autres groupes affiliés remettent en question le financement de leur organisme parapluie et son rôle de porte-parole, tout en désirant demeurer membre de cet organisme parapluie et chercher à sauvegarder ses acquis.

\section{Stratégies particulières}

Plusieurs stratégies particulières ont été employées afin de promouvoir notre vision féministe à l'intérieur de la Coalition et concrétiser les objectifs d'équité et de parité défendus par la Table féministe. En voici deux exemples.

Le premier exemple a eu lieu en juin 1995, à l'occasion du second forum communautaire. Lors de cette rencontre, nous avions 
à choisir un nom servant à désigner la Coalition. Certaines associations représentant des personnes de minorités visibles souhaitaient que le nom comprenne une référence explicite aux «minorités raciales francophones». Bien que cette expression puisse être perçue comme étant réductrice, la Table féministe a jugé bon, dans les circonstances, d'appuyer l'appellation «Coalition pour le développement et l'épanouissement de la communauté francoontarienne et des minorités raciales francophones de l'Ontario", et ce dans le but d'inclure symboliquement et de promouvoir cette réalité en Ontario français ${ }^{11}$. La stratégie utilisée par les femmes a donc été de s'allier aux groupes noirs francophones qui revendiquaient cette appellation et de mettre un terme à la recherche d'un autre nom désignant la "communauté francoontarienne» ${ }^{12}$. Une telle alliance n'a pas reçu l'assentiment de tous puisque certaines représentantes et certains représentants de groupes désiraient seulement retenir l'appellation francoontarienne pour qualifier la Coalition. Au départ, il faut dire que les femmes penchaient davantage pour l'expression «communautés francophones de l'Ontario" pour exprimer la diversité des groupes en présence. Or, à notre grande surprise, cette appellation n'a pas fait l'unanimité.

Le second exemple fait lui aussi référence à ce troisième forum. Il correspond au moment où nous avons réussi à faire adopter le vote sur la parité. La façon dont s'est déroulé l'acceptation de la clause sur la parité hommes/femmes dans le texte de l'Entente fut la suivante. Les groupes de minorités raciales ont exigé une représentation statutaire pour leur groupe respectif. En même temps, les femmes en demandaient autant pour elles. Profitant de ces deux demandes, la représentante de la Table féministe a alors fait la proposition que le nombre total de sièges à chacune des instances décisionnelles soit réparti de la façon suivante: un tiers octroyé à la représentation des femmes, un tiers octroyé aux minorités raciales et un tiers pour l'ensemble des autres groupes. On peut facilement imaginer le tollé et la consternation qu'une telle proposition a soulevé dans la salle. Les réactions n'ont pas tardé et les tractations en coulisse aussi. Quelques minutes ont 
suffi pour qu'une contre-proposition soit présentée. Cette contreproposition offrait, pour toutes les instances décisionnelles de la Coalition et de l'Entente, la parité de sièges entre les hommes et les femmes, et réservait deux sièges aux minorités raciales ${ }^{13}$. La présidence a alors demandé l'opinion des groupes de minorités raciales qui ont accepté cette contre-proposition. Nous lui avons aussi accordé notre appui et avons retiré notre proposition initiale. Le tout était joué.

\section{En conclusion, les défis à relever...}

"...les défis qui se posent au mouvement féministe demeurent encore ceux de l'équité, de la parité et du leadership transversal.»

\section{Défis au quotidien}

Dans la dynamique du milieu associatif francophone, les défis qui se posent au mouvement féministe demeurent encore ceux de l'équité, de la parité et du leadership transversal. Nous devons prendre la parole dans les divers espaces publics et politiques en dénonçant le plus souvent possible les inégalités et les iniquités sur les plans politique, social ou économique. Par ailleurs, la Table féministe aurait avantage à développer et à promouvoir son modèle de leadership transversal dans les milieux où elle intervient. Aux yeux des représentantes de la Table féministe, l'émergence de nouveaux leaders en Ontario français est déjà le signe avantcoureur de la validité de ce modèle transversal de représentation et de leadership.

\section{Défis spécifiques à l'Entente Canada-Communauté, région Ontario}

L'expérience de participation de la Table féministe à la Coalition soulève un certain nombre de défis au chapitre de l'affirmation politique des femmes.

Depuis la signature de l'Entente, soit le 13 décembre 1996, la Coalition n'existe plus. Elle a été remplacée par un Comité de démarrage qui verra à la mise sur pied d'un comité conjoint devant 
"La Table féministe a toujours eu comme objectif de prendre une part active au débat public, et notamment de débattre la question du leadership en Ontario français.» gérer l'Entente ${ }^{14}$. La Table féministe a refusé de signer l'Entente et de participer à sa gestion ${ }^{15}$.

Bien que nous ayons obtenu la parité et l'équité dans l'Ententecadre, plusieurs éléments demeuraient dans l'ombre et ne nous satisfaisaient pas. C'est le cas notamment des axes de développement imposés par Patrimoine Canada, du plan de développement des minorités raciales élaboré par trois associations seulement, des outils et des mécanismes d'évaluation de gestion de l'Entente qui demandaient encore à être définis et d'un montant obscur de 4,3 millions de dollars devant provenir d'une autre enveloppe budgétaire. De plus, nous refusions de gérer la décroissance de l'enveloppe budgétaire initialement prévue avant la signature de l'Entente ${ }^{16}$. Mentionnons qu'à la signature de l'Entente Canada-Communauté, région Ontario, en 1996, laquelle est valide pour une durée de trois ans, il ne reste plus que 3,5 millions de dollars à gérer et de cette somme, 1,2 millions de dollars ont déjà été consentis aux ACFO provinciale et régionales ${ }^{17}$.

Il y a des avantages et des désavantages liés à notre refus de signer l'Entente, mais là n'est pas l'objet de cet article. La Table féministe a toujours eu comme objectif de prendre une part active au débat public, et notamment de débattre la question du leadership en Ontario français. Comme partie intégrante de l'Entente Canada-Communauté, région Ontario, nous croyons que la réflexion entreprise sur la représentation politique et le leadership au sein de la Coalition aura permis d'amener ce débat sur la place publique.

Chose certaine, la Ministre de Patrimoine Canada, en tant que ministre responsable de l'Entente-cadre, doit s'assurer que les principes de reconnaissance de la diversité et de l'iniquité historique subie par les francophones et les femmes et reconnues dans la Loi sur les langues officielles et le Plan fédéral pour l'égalité entre les sexes, soient mises en vigueur ${ }^{18}$. En raison des élections fédérales de juin 1997, le cabinet de la Ministre nous a invité à nous inscrire dans la démarche prévue par l'Entente.Au lendemain des élections, nous chercherons donc à poursuivre la discussion auprès de la Ministre et de ses conseillers afin de pousser plus loin nos revendications en matière de représentativité et d'équité pour 
que nos communautés soient bien outillées et puissent assurer leur développement et leur plein épanouissement.

\section{Notes}

1. Cet article est issue d'une communication présentée au colloque Visibles et Partenaires. Des circonstances exceptionnelles ont empêché l'auteure de présenter cette comunication qui a été lue par Blanche Paradis. Je tiens à la remercier. Les propos de cette article n'engagent que l'auteure. Je tiens aussi à remercier Andrée Côté, Chantal Cholette, Geneviève Guindon, Guylaine Leclerc, Blanche Paradis et, tout particulièrement, Linda Cardinal pour leur appui à la rédaction de cette communication.

2. Extrait de la trousse de promotion de la Table.

3. Par exemple, celui du Comité des femmes pour l'éducation (AÉFO_.

4. Un forum communautaire a eu lieu en décembre 1994. C'est alors que la base de la nouvelle entente a été fixée, c'est-à-dire une entente de cogestion et ce qu'elle comprend: les programmes d'appui, la concertation ministérielle et intraministérielle.

5. Nous pensons ici au rôle prédominant qu'a joué l'ACFO provinciale comme principal porte-parole de la communauté franco-ontarienne.

6. Extrait de l'Entente Canada-Communauté, annexe 5B.

7. À l'exception de la Table féministe francophone de concertation provinciale et de l'Alliance culturelle de l'Ontario qui fonctionnent avec des structures et une représentation souples, les autres groupes avaient une structure plus conventionnnelle.

8. La première rencontre de la Table à l'exécutif de la Coalition a eu lieu le 21 septembre1994.

9. Comité de démarche politique, Reconceptualisation du réseau associatiffrancophone en Ontario, ébauche, Ottawa, mars 1997.

10. La Patente est une organisation secrète formée à la suite du Règlement 17 pour défendre et promouvoir le fait français au Canada. Elle est aussi connue sous le nom de l'Ordre de Jacques-Cartier.

11. En effet, ce terme exclut, à mon avis, les personnes d'une communauté francophone sur une base autre que la race.

12. J'exclus ici les associations de femmes noires, africaines ou ethnoculturelles, membres de la Table féministe.

13. La représentation des minorités raciales est aussi soumise au principe de la parité entre les hommes et les femmes.

14. Voir l'annexe 5B de l'Entente. 
15. Trente-huit des 70 groupes ayant droit de vote à la Coalition, soit $54 \%$, se sont prononcées en faveur de l'Entente.

16. Lettre adressée le 18 décembre 1996 à l'Honorable Sheila Copps, ministre de Patrimoine Canada.

17. Bien que l'Entente conclue approche les 18 millions de dollars et couvre la période allant de 1994 à 1997, les sommes restantes au moment de la signature de l'Entente équivalent en gros à 3,5 millions de dollars. Ces sommes apparaissent dans le compterendu de la réunion de la Table féministe de concertation provinciale de l'Ontario, tenue à Ottawa en février 1996, pages 7 à 10.

18. Lettre de la Table féministe adressées à la Ministre Sheila Copps en date du 18 décembre 1996. 